\title{
ITÔ'S THEOREM ON GROUPS WITH TWO CLASS SIZES REVISITED
}

\author{
ELENA ALEMANY ${ }^{\bowtie}$, ANTONIO BELTRÁN and MARÍA JOSÉ FELIPE
}

(Received 30 June 2011)

\begin{abstract}
Let $G$ be a finite $p$-solvable group. We prove that if $G$ has exactly two conjugacy class sizes of $p^{\prime}$-elements of prime power order, say 1 and $m$, then $m=p^{a} q^{b}$, for two distinct primes $p$ and $q$, and $G$ either has an abelian $p$-complement or $G=P Q \times A$, with $P$ and $Q$ a Sylow $p$-subgroup and a Sylow $q$-subgroup of $G$, respectively, and $A$ is abelian. In particular, we provide a new extension of Itô's theorem on groups having exactly two class sizes for elements of prime power order.
\end{abstract}

2010 Mathematics subject classification: primary 20E45; secondary 20D20.

Keywords and phrases: finite groups, conjugacy class sizes, solvable groups.

\section{Introduction}

A classic theorem of Itô asserts that any finite group $G$ having exactly two class sizes is a nilpotent group and more precisely, is a direct product of a $p$-group and an abelian $p^{\prime}$-group. Later, Isaacs provided a new and simpler proof of this theorem by using some results on abelian partitions (see [6]).

Some authors replace conditions for all conjugacy classes by conditions referring to only some of the classes in order to investigate the structure of a finite group; for instance, the conjugacy classes of elements of prime power order or $p^{\prime}$-elements for some prime $p$. Results of this type are interesting since they can be used to simplify the proofs of new or known properties related to conjugacy classes. Baer proves in [1] that a group $G$ is solvable if the class sizes of its elements of prime power order are also prime powers. In 1996, Shirong showed that any group $G$ having exactly two class sizes of elements of prime power order must be solvable [9]. The proof relies on certain consequences of the classification of the finite simple groups and on the Feit-Thompson theorem on the solvability of a group of odd order. Recently, by using the same arguments as in [9], Zhao and Guo [10] have proved the solvability

This research is supported by the Spanish Government, Proyecto MTM2010-19938-C03-02 and the second and third authors are supported by the Valencian Government, Proyecto PROMETEO/2011/30. The second author is also supported by grant Fundació Caixa-Castelló P11B2010-47.

(C) 2011 Australian Mathematical Publishing Association Inc. 0004-9727/2011 \$16.00 
of a $p$-solvable group with exactly two class sizes of $p^{\prime}$-elements of prime power order. Other extensions of Itô's theorem study the structure of a group $G$ with two class sizes of $p^{\prime}$-elements. Camina showed in [4] that such groups are solvable, by using the description of finite groups with dihedral Sylow 2-subgroups given by Gorenstein and Walter. This demonstrates the difficulties that arise when one studies the structure of groups from the class sizes of its primary elements or its $p^{\prime}$-elements.

In [2], Beltrán and Felipe proved that a $p$-solvable group $G$ with two class sizes of $p^{\prime}$-elements either has abelian $p$-complements or is a $\{p, q\}$-group, up to central factors. In this note, we extend the latter result on groups with two class sizes of $p^{\prime}-$ elements of prime power order, proving that such groups have the same structure, but we remark that the approach is completely different. The proof in [2] is based on the fact that the centralizers of $p^{\prime}$-elements are nilpotent and have abelian $p$-complement, while this property does not hold when we consider the hypothesis on class sizes only on the elements of prime power order.

Theorem A. Suppose that $G$ is a finite p-solvable group and that 1 and $m$ are the conjugacy class sizes of $p^{\prime}$-elements of prime power order. Then $m=p^{a} q^{b}$, with $q a$ prime distinct from $p$, and $a, b \geq 0$. If $b=0$, then $G$ has abelian $p$-complements. If $b \neq 0$, then $G=P Q \times A$, with $P$ and $Q$ a Sylow $p$-subgroup and a Sylow $q$-subgroup of $G$, respectively, and $A \leq \mathbf{Z}(G)$.

In particular, we obtain the nilpotency of groups with two class sizes of elements of prime power order, extending Itô's theorem while improving Shirong's result.

Corollary B. Let $G$ be a finite group and suppose that $G$ has exactly two class sizes of elements of prime power order, 1 and $m$. Then $m$ is a prime power and $G$ is nilpotent. Moreover, $G=Q \times A$, with $Q$ a Sylow q-subgroup of $G$ and $A \leq \mathbf{Z}(G)$.

Notice that the set of elements considered is likely to be the most reduced possible one, for if we weaken the hypothesis and work only with elements of prime order the thesis of Itô's theorem is false. It is enough to consider a group $G$ defined as the semidirect product of the quaternion group of order eight acted on nontrivially by a cyclic group of order three. All elements of prime order of $G$ have class size 1 or 6 , while $G$ is trivially nonnilpotent.

\section{Proofs}

All groups considered are finite. The conjugacy class of an element $x \in G$ is denoted by $x^{G}$, and its size is called the index of $x$ in $G$. We need some elementary results.

Lemma 2.1. Let $G$ be a group. A prime $p$ does not divide any conjugacy class size of $G$ if and only if $G$ has a central Sylow p-subgroup.

Proof. See for instance [6, Theorem 33.4].

The following extends for an arbitrary set of primes $\pi$ and for $\pi$-separable groups a theorem of Camina and Camina (an immediate consequence of [5, Proposition 1]), which asserts that any element of index $p^{a}$ in a group $G$ must lie in $\mathbf{O}_{p, p^{\prime}}(G)$. 
Lemma 2.2. Let $G$ be a $\pi$-separable group.

(a) If $x \in G$ and $\left|x^{G}\right|$ is a $\pi$-number, then $x \in \mathbf{O}_{\pi, \pi^{\prime}}(G)$.

(b) The conjugacy class size of any $\pi^{\prime}$-element in $G$ is a $\pi$-number if and only if $G$ has abelian Hall $\pi^{\prime}$-subgroups.

Proof. This is [3, Theorem C] and part (b) is [3, Lemma 7].

Theorem 2.3. Let $P \times Q$ be a direct product of a p-group $P$ and a $p^{\prime}$-group $Q$ represented as a group of automorphisms of a p-group $G$. Suppose that $\mathbf{C}_{G}(P) \subseteq$ $\mathbf{C}_{G}(Q)$. Then $Q$ acts trivially on $G$.

Proof. This is Thompson's $P \times Q$-lemma. See, for instance, [8, 8.2.8].

We will first prove Theorem A for solvable groups.

THeOREM 2.4. Let $G$ be a solvable group and suppose that $G$ has exactly two class sizes of $p^{\prime}$-elements of prime power order, 1 and $m$. Then $m=p^{a} q^{b}$, with $q$ a prime distinct from $p$, and $a, b \geq 0$. If $b=0$, then $G$ has abelian $p$-complements. If $b \neq 0$, then $G=P Q \times A$, with $P$ and $Q$ a Sylow p-subgroup and a Sylow q-subgroup of $G$, respectively, and $A \leq \mathbf{Z}(G)$.

Proof. We proceed by induction on $|G|$. First we will show that any prime $r \neq$ $p$ dividing $|G|$, also divides $m$. Suppose that $r \neq p$ is a prime which does not divide $m$. Then the class size of every $p^{\prime}$-element of prime power order is an $r^{\prime}$-number, so by Lemma 2.2, we have $x \in \mathbf{O}_{r^{\prime}, r}(G)$. This implies that we can write $G=P \mathbf{O}_{r^{\prime}, r}(G)$, where $P$ is a Sylow $p$-subgroup of $G$. Let $K=P \mathbf{O}_{r^{\prime}}(G)$. Notice that $\mathbf{O}_{r^{\prime}}(G)$ cannot be central in $G$, otherwise $m$ would be a $p$-power, and then, by Lemma 2.2, the $p$ complements of $G$ would be abelian and the theorem would be proved. Thus, if $y$ is a $p^{\prime}$-element of prime power order of $K$, which is noncentral in $G$, as $|G: K|$ is an $r$-number, then $G=\mathbf{C}_{G}(y) K$ and we have $m=\left|K: \mathbf{C}_{K}(y)\right|$. By applying the inductive hypothesis, we get that either $m=p^{a}$ and $K$ has abelian $p$-complements or $m=p^{a} q^{b}$ for some prime $q \neq p, b>0$ and $K=P Q \times A$, with $P \in \operatorname{Syl}_{p}(G), Q \in \operatorname{Syl}_{q}(G)$ and $A$ is abelian.

Suppose first that $m=p^{a}$ and $K$ has an abelian $p$-complement. Applying Lemma 2.2, we get that every $p^{\prime}$-element of $G$ of prime power order belongs to $N:=\mathbf{O}_{p, p^{\prime}}(G)$. As a consequence, every $p^{\prime}$-element of $G$ lies in $N$ too. Write $N=\mathbf{O}_{p}(G) H$, with $H$ a $p$-complement of $G$. If $x \in H$ has prime power order, then

$$
\begin{aligned}
\left|G: \mathbf{C}_{G}(x) \| \mathbf{C}_{G}(x): \mathbf{C}_{N}(x)\right| & =|G: N|\left|N: \mathbf{C}_{N}(x)\right| \\
& =|G: N|\left|\mathbf{O}_{p}(G): \mathbf{C}_{\mathbf{O}_{p}(G)}(x) \| H: \mathbf{C}_{H}(x)\right| .
\end{aligned}
$$

Since $\left|\mathbf{C}_{G}(x): \mathbf{C}_{N}(x)\right|=\left|\mathbf{C}_{G}(x) N: N\right|$ and $\left|G: \mathbf{C}_{G}(x)\right|$ are $p$-numbers, it follows that $\left|H: \mathbf{C}_{H}(x)\right|=1$. Hence $H$ is abelian and $G$ has abelian $p$-complements, as wanted.

Now, assume the second possibility, that is, $K=P Q \times A$, and $m=p^{a} q^{b}$, with $q \neq p$ and $b>0$. We prove that $A$ is central in $G$. If $w \in A$ is a noncentral element of prime power order, then $Q \subseteq \mathbf{C}_{G}(w)$. This contradicts the fact that $m=\left|G: \mathbf{C}_{G}(w)\right|$ and $q$ does 
divide $m$. Therefore, by Lemma 2.1 and by induction we can assume that $K$ is a $\{p, q\}$ group (and $G$ a $\{p, q, r\}$-group). Now, we write $\mathbf{O}_{r^{\prime}}(G)=P_{0} Q$ for some $p$-subgroup $P_{0}$ and $Q$ a Sylow $q$-subgroup of $G$. Notice that if $H$ is a $p$-complement of $G$ such that $Q \subseteq H$, then $H \cap \mathbf{O}_{r^{\prime}}(G)=Q \unlhd H$, so we conclude that every $p$-complement of $G$ has a normal Sylow $q$-subgroup. We can suppose that there exists some noncentral $r$-element $x \in G$, otherwise the theorem is proved. Let $Q_{0}$ be a Sylow $q$-subgroup of $\mathbf{C}_{G}(x)$ and let $H$ be a $p$-complement of $G$ such that $\left\langle Q_{0}, x\right\rangle \subseteq H$. Let us consider the action of $Q_{0} \times\langle x\rangle$ on the (normal) Sylow $q$-subgroup $Q$ of $H$. We claim that $\mathbf{C}_{Q}\left(Q_{0}\right) \subseteq Q_{0}$. Let $z \in \mathbf{C}_{Q}\left(Q_{0}\right)$. If $z$ is central in $G$, then trivially $z \in \mathbf{Z}(G)_{q} \subseteq Q_{0}$, and if $z \notin \mathbf{Z}(G)$, then $\left\langle Q_{0}, z\right\rangle \subseteq \mathbf{C}_{G}(z)$. However, $\left|\mathbf{C}_{G}(z)\right|_{q}=\left|Q_{0}\right|$, and consequently, $z \in Q_{0}$. We apply Theorem 2.3 and conclude that $x \in \mathbf{C}_{G}(Q)$, that is, $Q \subseteq \mathbf{C}_{G}(x)$, which contradicts the fact that $q$ divides $m$.

Therefore, for the rest of the proof we will assume that any prime $r$ divisor of $|G|_{p^{\prime}}$ also divides $m$. Now we are going to work in the factor group $\bar{G}=G / \mathbf{O}_{p}(G)$. Let $x$ be a noncentral $p^{\prime}$-element of $G$ of prime power order. Then we can write $\mathbf{C}_{\bar{G}}(\bar{x})=H_{0} P_{0} / \mathbf{O}_{p}(G)$, with $P_{0}$ a $p$-subgroup, $H_{0}$ a $p$-complement of $H_{0} P_{0}$ and $x \in H_{0}$. If $y \in H_{0}$, then $\bar{y} \in \mathbf{C}_{\bar{G}}(\bar{x})$ and so $[x, y] \in H_{0} \cap \mathbf{O}_{p}(G)=1$. Thus we have

$$
H_{0} \subseteq \mathbf{C}_{G}(x) \subseteq H_{0} P_{0} \subseteq G
$$

Therefore $\left|\bar{G}: \mathbf{C}_{\bar{G}}(\bar{x})\right|_{p^{\prime}}=\left|G: H_{0} P_{0}\right|_{p^{\prime}}=m_{p^{\prime}}$ and $\left|\mathbf{C}_{\bar{G}}(\bar{x})\right|_{p^{\prime}}=|\bar{G}|_{p^{\prime}} / m_{p^{\prime}}$ is constant. Now we prove that $\overline{\mathbf{Z}(G)} \bar{p}^{\prime}=\mathbf{Z}(\bar{G})_{p^{\prime}}$. The direct inclusion is trivial, so we take a central $s$-element $\bar{x}$ of $\bar{G}$, for some prime $s \neq p$. Then, by considering the $\left\{s, s^{\prime}\right\}$ decomposition of $x$, we can assume that $x$ is an $s$-element of $G$. Suppose that $x \notin \mathbf{Z}(G)$. By applying the above equality, we have $1=\left|\bar{G}: \mathbf{C}_{\bar{G}}(\bar{x})\right|_{p^{\prime}}=m_{p^{\prime}}$, and so $m$ is a power of $p$, which is clearly a contradiction. Consequently, $x \in \mathbf{Z}(G)_{p^{\prime}}$ and the equality is proved.

Since $\bar{G}$ is solvable (and nonabelian) and $\mathbf{O}_{p}(\bar{G})=1$, there exists a prime $q \neq p$ such that $\mathbf{Z}(\bar{G})_{q}<\mathbf{O}_{q}(\bar{G})$. Assume that $\bar{G}$ has a noncentral $r$-element $\bar{w}$, for some prime $r \notin\{p, q\}$. As before, $w$ can be assumed to be a noncentral $r$-element of $G$. Let $\overline{Q_{0}}$ be a Sylow $r$-subgroup of $\mathbf{C}_{\bar{G}}(\bar{w})$. We consider the action of $\overline{Q_{0}} \times\langle\bar{w}\rangle$ on $\bar{Q}:=\mathbf{O}_{q}(\bar{G}) \overline{Q_{0}}$ and we claim that $\mathbf{C}_{\bar{Q}}\left(\overline{Q_{0}}\right) \subseteq \overline{Q_{0}}$. If $\bar{z} \in \mathbf{C}_{\bar{Q}}\left(\overline{Q_{0}}\right)$ is a noncentral $q$-element of $\bar{G}$, then $z$ can be assumed to be a noncentral $q$-element of $G$, and so $\left\langle\overline{Q_{0}}, \bar{z}\right\rangle \subseteq \mathbf{C}_{\bar{G}}(\bar{z})$. However, as we have proved before,

$$
\left|\mathbf{C}_{\bar{G}}(\bar{z})\right|_{q}=|\bar{G}|_{q} / m_{q}=\left|\mathbf{C}_{\bar{G}}(\bar{w})\right|_{q}=\left|\overline{Q_{0}}\right|,
$$

and consequently $\bar{z} \in \overline{Q_{0}}$. Applying now Theorem 2.3 we obtain that $\bar{w} \in \mathbf{C}_{\bar{G}}(\bar{Q})$, and so $\bar{w} \in \mathbf{C}_{\bar{G}}\left(\mathbf{O}_{q}(\bar{G})\right)$. As $\overline{\mathbf{Z}(G)_{p^{\prime}}}=\mathbf{Z}(\bar{G})_{p^{\prime}}$, this property holds for every $r$-element $w$ in $G$. Therefore, if $R$ is a Sylow $r$-subgroup of $G$ then $\overline{R \mathbf{O}_{p}(G)} \subseteq \mathbf{C}_{\bar{G}}\left(\mathbf{O}_{q}(\bar{G})\right)$. On the other hand, there exists $\bar{t} \in \mathbf{O}_{q}(\bar{G}) \backslash \mathbf{Z}(\bar{G})$, such that $\overline{R \mathbf{O}_{p}(G)} \subseteq \mathbf{C}_{\bar{G}}(\bar{t}) \subset \bar{G}$, which is clearly a contradiction, since $r$ divides $m_{p^{\prime}}=\left|\bar{G}: \mathbf{C}_{\bar{G}}(\bar{t})\right|_{p^{\prime}}$. 
This contradiction implies that $|\bar{G}: \mathbf{Z}(\bar{G})|$ is a $\{p, q\}$-number, and hence $|G: \mathbf{Z}(G)|$ is a $\{p, q\}$-number too. As we have proved that any prime $q \neq p$ dividing $|G|$ also divides $m$, we conclude that $G$ is a $\{p, q\}$-group and the theorem is proved.

Now we can prove the main results in the introduction.

Proof of Theorem A. By the main theorem of [10], we have that $G$ is solvable and then we apply Theorem 2.4.

As a particular case, we can get a new extension of Itô's theorem on groups with two class sizes for elements of prime power order.

Proof of Corollary B. By a theorem of Shirong [9] $G$ is solvable. Let us take a prime $p$ which does not divide the order of $G$. Then, all $p^{\prime}$-elements of prime power order of $G$ have class size 1 or $m$. By Theorem A, we have that $m=q^{b}$ and $G=Q \times A$, with $Q \in \operatorname{Syl}_{q}(G)$ and $A \leq \mathbf{Z}(G)$.

Remark. As a consequence of Corollary B, when a group $G$ has two class sizes of elements of prime power order, then all elements in $G$ have only two class sizes too.

\section{Acknowledgement}

This work is part of the first author's $\mathrm{PhD}$ thesis at the Universidad Politécnica de Valencia.

\section{References}

[1] R. Baer, 'Group elements of prime power index', Trans. Amer. Math. Soc. 75 (1953), 20-47.

[2] A. Beltrán and M. J. Felipe, 'Finite groups with two $p$-regular conjugacy class lengths', Bull. Aust. Math. Soc. 67 (2003), 163-169.

[3] A. Beltrán and M. J. Felipe, 'Prime powers as conjugacy class lengths of $\pi$-elements', Bull. Aust. Math. Soc. 69 (2004), 317-325.

[4] A. R. Camina, 'Finite groups of conjugate rank 2', Nagoya Math. J. 53 (1974), 47-57.

[5] A. R. Camina and R. D. Camina, 'Implications of conjugacy class size', J. Group Theory 1 (1998), 257-269.

[6] B. Huppert, Character Theory of Finite Groups, De Gruyter Expositions in Mathematics, 25 (Walter De Gruyter, New York, 1998).

[7] N. Itô, 'On finite groups with given conjugate type I', Nagoya Math. J. 6 (1953), 17-28.

[8] K. Kurzweil and B. Stellmacher, The Theory of Finite Groups. An Introduction (Springer, New York, 2004).

[9] L. Shirong, 'Finite groups with exactly two class lengths of elements of prime power order', Arch. Math. (Basel) 67 (1996), 100-105.

[10] X. Zhao and X. Guo, 'On conjugacy class sizes of the $p^{\prime}$-elements with prime power order', Algebra Colloq. 16(4) (2009), 541-548.

ELENA ALEMANY, Departamento de Matemática Aplicada, Universidad Politécnica de Valencia, 46022 Valencia, Spain

e-mail: ealemany@mat.upv.es 
ANTONIO BELTRÁN, Departamento de Matemáticas, Universidad Jaume I, 12071 Castellón, Spain

e-mail: abeltran@mat.uji.es

MARÍA JOSÉ FELIPE, Instituto Universitario de Matemática Pura y Aplicada, Universidad Politécnica de Valencia, 46022 Valencia, Spain

e-mail:mfelipe@mat.upv.es 